Internist

2000 · 41: 970-984 @ Springer-Verlag 2000
Übersicht

E. Meissner · M. Hamm · H. Fabel

Abteilung Pneumologie der Medizinischen Hochschule Hannover

\section{Zum Thema}

Die nicht-invasive Beatmung (NIV) über eine Maske ermöglicht bei vielen Patienten eine effiziente Beatmung unter Vermeidung der Risiken und Nebenwirkungen der endotrachealen Intubation. Für die chronisch-obstruktive Lungenerkrankung (COPD) sind die Vorteile der Maskenbeatmung im Hinblick auf Beatmungsdauer, Dauer der Intensivbehandlung, Prognose und Behandlungskosten inzwischen relativ gut durch Studien belegt. Bei anderen Indikationen - „NichtCOPD-Patienten" - erlauben die verfügbaren Daten noch kein gesichertes Urteil, rechtfertigen aber einen Behandlungsversuch auch außerhalb von Studien in klinischer Routine. Nachteilig sind der initial höhere personelle Betreuungsaufwand bei NIV sowie die stark von der Erfahrung abhängige Erfolgsrate.

Die wichtigste Voraussetzung für eine erfolgreiche nicht-invasive Beatmung in der Intensivmedizin ist die Bereitschaft von Ärzten und Pflegepersonal, eine ungewohnte Beatmungsphilosophie zu akzeptieren und die relativ einfache Technik der Maskenbeatmung zu erlernen. Bei Beherrschung der Technik kann die NIV aber eine wertvolle Hilfe vom Notarztwagen bis zur Intensivstation sein.

\section{Schlüsselwörter}

Nicht-invasive Beatmung · Maskenbeatmung Akute respiratorische Insuffizienz . Intensivstation · Intensivmedizin ie akute respiratorische Insuffizienz erfordert bei schwerer Ausprägung eine mechanische Beatmung. Üblicherweise wird die Beatmung über einen endotrachealen Tubus als Zugangsweg durchgeführt. Die endotracheale Intubation ist sowohl akut als auch chronisch mit Problemen und Komplikationen belastet. Während akut Verletzungen der Atemwege bei der Intubation, Verlust von Schluckvermögen, Sprache, Hustenstoß sowie Schleimhautschädigungen auftreten können, sind bei länger dauernder Intubation erhöhte Infektionsgefahr und nach Extubation - Stimmbandparesen, Trachealstenosen und -malazie zu nennen. Eine Alternative ist daher die nicht-invasive Beatmung (Non-invasive Ventilation, NIV) über eine Maske.

Die nicht-invasive Beatmung ist in der Heimbeatmung gut etabliert und eine weit angewandte Technik. Anders sieht das auf der Intensivstation (ICU) aus, obwohl inzwischen eine Reihe von Studien den sinnvollen Einsatz dieser Technik auf der ICU belegen. Trotzdem ist die Akzeptanz der NIV bei Intensivmedizinern bisher gering. Sie ist meist nur in den Häusern auf der ICU eingeführt, die ein Schlaflabor betreiben und so Kontakt zu der Technik haben.

Für diese Entwicklung gibt es mehrere Ursachen. Grundsätzlich ist mit den „großen“ Beatmungsgeräten eine Maskenbeatmung durchzuführen. Dabei können eine Reihe von Problemen auftreten. Unter anderem kann sich der Patient mit einer an das übliche Schlauchsystem angeschlossenen Maske wegen der relativ rigiden Schläuche nur schlecht bewegen. Optimal ist der Einsatz kleine- rer Beatmungsgeräte - z. B. der Firmen Breas, Healthdyne, Dräger u. a. - die mit entsprechenden Schlauchsystemen geliefert werden. Bisher gibt es aber nur wenig Kontakte zwischen den Herstellern der „kleinen“ Beatmungsgeräte und Intensivmedizinern.

Probleme der ,kleinen“ Beatmungsgeräte für Maskenbeatmung sind die teilweise andere Bedienung und die teilweise nicht identischen Parameter. So wird z. B. in der Intensivmedizin das Atemzugvolumen üblicherweise exspiratorisch gemessen, während bei den Geräten für Maskenbeatmung - aufgrund des fehlenden Exspirationsschlauchs in der Regel nur das inspiratorische Atemzugvolumen gemessen und angezeigt wird.

Letztlich ist auch das Management ein Problem. Bei der invasiven Beatmung wird der Patient sediert, relaxiert, intubiert und beatmet. Bei der Einleitung einer Maskenbeatmung müssen sich Ärzte und Pflegepersonal mindestens in den ersten 1-2 $h$ intensiv um den Patienten kümmern, bis die Tolerierung der Maskenbeatmung erreicht ist.

\section{Nicht-invasive versus invasive Beatmung}

Vorteil der NIV ist das Aufrechterhalten physiologischer Verhältnisse (Tabelle 1). Die Patienten können weiter sprechen,

\section{Dr. E. Meissner}

Abteilung Pneumologie, Medizinische Hochschule Hannover, Carl-Neuberg-Straße 1, 30625 Hannover,

E-Mail:eckehard.meissner@t-online.de 


Tabelle 1

Vor- und Nachteile invasiver vs. nicht-invasiver Beatmung

\begin{tabular}{|c|c|c|}
\hline & Invasiv & Nicht-invasiv \\
\hline Kooperation des Patienten & Nicht notwendig & Notwendig \\
\hline Beatmungszugang & Sicher & Unsicher \\
\hline \multicolumn{3}{|l|}{ Beatmung } \\
\hline Einleitung & Leicht & Schwierig \\
\hline Unterbrechung & Nicht möglich & Leicht möglich \\
\hline Beendigung & Häufig schwierig & Leicht \\
\hline Beatmungseinstellungen & Frei wählbar, auch „unphysiologische“ Muster möglich & Keine „unphysiologischen“ Muster möglich \\
\hline Beatmungsparameter & Einhaltung garantiert & Einhaltung nicht garantiert (Leckage!) \\
\hline Beatmungsmonitoring & Zuverlässig & Nur eingeschränkt \\
\hline Analgosedierung & Notwendig & Allenfalls minimale Sedierung \\
\hline Atemwege & Geschützt & Ungeschützt \\
\hline Bronchialtoilette & Einfach & Schwierig \\
\hline Aspiration & Relativer Schutz & Aerophagie, Aspirationsgefahr \\
\hline Komplikationen & $\begin{array}{l}\text { Früh-/Spätkomplikationen durch Intubation } \\
\text { und Tubus (Strikturen etc.), Infektionen }\end{array}$ & $\begin{array}{l}\text { Maskenkomplikationen (Hautnekrosen, } \\
\text { Leckage, Konjunktivitis), Aerophagie, Aspiration } \\
\text { Keine Spätkomplikationen }\end{array}$ \\
\hline Nosokomiale Infektionen & Risiko erhöht & Risiko geringer \\
\hline Komplikationsrate & & $\begin{array}{l}\text { Reduziert (?) } \\
\text { Krankenhausaufenthalt? } \\
\text { Mortalität? }\end{array}$ \\
\hline Sprechen & Nicht möglich & Erhalten \\
\hline Schlucken & Nur eingeschränkt möglich & Physiologisch möglich \\
\hline Hustenstoß & Nur eingeschränkt möglich & Physiologisch möglich \\
\hline Enterale Ernährung & $\begin{array}{l}\text { Nur eingeschränkt möglich (Darmatonie } \\
\text { durch Sedativa!) }\end{array}$ & Oral, in Beatmungspausen möglich \\
\hline Mobilisierung & Bedingt möglich & Möglich \\
\hline Personalaufwand & Bei Dauerbeatmungen höher & Initial höher \\
\hline Kosten & Hohe Kosten & Kostenreduktion (?) \\
\hline
\end{tabular}

schlucken sowie in Beatmungspausen essen und husten. Die Mobilisierung ist jederzeit möglich. Die Beatmung kann jederzeit unterbrochen werden. Bei Beendigung der NIV tritt keine WeaningPhase auf.

Gegenüber einer Intubation mit Beatmung sind der verminderte Medikamentenbedarf zur Analgosedierung sowie ein geringeres Verletzungsrisiko der oberen Atemwege als Vorteile zu nennen. Diskutiert werden darüber hinaus eine Verminderung intubationsspezifischer Komplikationen wie Atemwegsinfektionen oder Barotraumata, kürzere Krankenhausaufenthalte sowie niedrigere Mortalität. Hier ist die Datenlage aus Studien bisher für eine endgültige Beurteilung nicht ausreichend.

Nachteilig ist zunächst bei der Einleitung einer NIV, dass mehr Zeit als für eine endotracheale Intubation benötigt wird, um den Patienten an die Maske $\mathrm{zu}$ adaptieren. Meist $\mathrm{zu}$ behebende Maskenprobleme wie Hautverletzungen, Irritationen der Augen und trockene Nasenschleimhaut treten häufiger auf. Problematischer sind größere Leckagen mit einer möglichen Verschlechterung der respiratorischen Insuffizienz sowie Aerophagie mit Magenüberblähung und Gefahr der Aspiration. Insgesamt ist eine aufwendigere Überwachung der Patienten unter NIV durch Ärzte und Pflege- personal notwendig, da auch die Beatmungsgeräte selbst wenige Optionen zum Monitoring bieten.

Die endotracheale Intubation bietet Vorteile bei schweren Gasaustauschstörungen wie Pneumonie oder ARDS („acute respiratory distress syndrome“), die unphysiologische Beatmungseinstellungen oder hohe Beatmungsdrücke erfordern, sowie dauerhaften Beatmungen $>48 \mathrm{~h}$.

\section{NIV - Indikationen und Kontraindikationen}

Voraussetzung für eine NIV ist ein wacher, spontan-atmender und kooperativer 
Tabelle 2

Indikationen und Kontraindikationen für eine nicht-invasive Beatmung

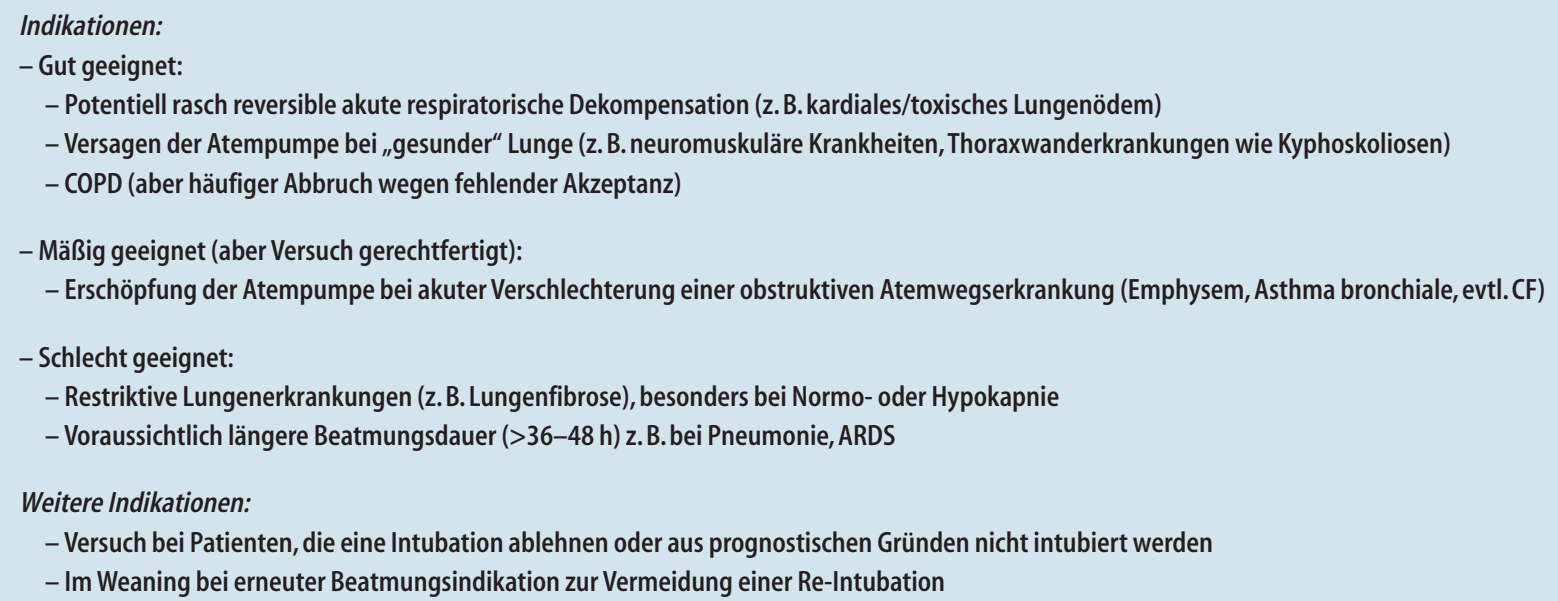

Patient, der keine „extrapulmonale“ Indikation für eine hochdosierte Sedativaoder Analgetikatherapie hat und keine „unphysiologischen“ Beatmungsmuster oder hohe Atemwegsdrucke benötigt.

Theoretisch kommen alle Patienten mit akuter respiratorischer Insuffizienz, die potentiell reversibel ist und eine Beatmungsdauer von bis $\mathrm{zu} 48 \mathrm{~h}$ erwarten lässt, in Betracht (Tabelle 2). Die Indikation zur NIV ist bei respiratorischer Dekompensation z. B. infolge kardialem oder toxischem Lungenödem (primär hypoxämisches Lungenversagen) gegeben [36]. Voraussetzung ist, dass der Patient kurze Beatmungspausen gut toleriert, kein PEEP („positive endexspiratory pressure") $>10 \mathrm{~cm} \mathrm{H}_{2} \mathrm{O}$ erforderlich ist und sich die klinische Situation wahrscheinlich innerhalb von 2 bis 3 Tagen bessert.

Ebenfalls gut geeignet ist ein hyperkapnisches Ventilationsversagen [33,36] infolge Versagen der Atempumpe bei "gesunder" Lunge wie z. B. bei neuromuskulären Krankheiten, Thoraxwanderkrankungen wie Kyphoskoliose sowie COPD. In diesen Fällen wird bei einer akuten Dekompensation die Beatmungsindikation unter dem Gesichtspunkt einer Dauerversorgung mit intermittierender häuslicher Beatmung gestellt.

Weniger geeignet ist - obwohl positive Erfahrungen berichtet werden und ein Versuch gerechtfertigt ist - eine Erschöpfung der Atempumpe bei akuter Verschlechterung einer obstruktiven Atemwegserkrankung wie Asthma bronchiale, Emphysem, evtl. CF (Cystische Fibrose, Mukoviszidose; $[6,8,9,36])$. Hier sollte die Indikation nur gestellt werden, wenn kurzfristig eine medikamentöse Besserung $\mathrm{zu}$ erhoffen ist oder unter Berücksichtigung der Gesamtsituation grundsätzlich der Versuch einer längerfristigen intermittierenden Beatmungstherapie geplant ist.

Schlecht geeignet - wenn auch zeitweise bei diesen Erkrankungen eingesetzt - sind restriktive Lungenerkrankungen (z. B. Lungenfibrose), besonders bei Normo- oder Hypokapnie. Auch eine voraussichtlich längere Beatmungsdauer (z. B. Pneumonie) spricht gegen NIV und für eine Intubation und konventionelle Beatmungstechnik.

Weitere Indikationen für eine NIV bestehen z. B. bei Patienten, die eine Intubation abgelehnt haben oder bei denen diese aus ethischen Gründen frag- lich indiziert ist [36] sowie bei erneuter Beatmungsindikation nach Extubation zur Vermeidung einer Re-Intubation $[28,36,52]$.

Kontraindikationen liegen bei völlig unkooperativen bzw. komatösen Patienten vor. Bei Bewusstlosigkeit - insbesondere infolge $\mathrm{CO}_{2}$-Narkose - ist ein Versuch möglich. Allerdings muss die Maske i.d.R. von Hand gehalten werden. Zusätzlich ist das Offenhalten der oberen Atemwege durch den EsmarchHandgriff notwendig. Andere Faktoren, die gegen NIV sprechen, sind Schock, schwere Herzrhythmusstörungen mit Hypotonie, kardiale Ischämien, Aspirationsgefahr z. B. infolge fehlender Schutzreflexe, Verlegung der oberen Atemwege, starke Verschleimung [51], Notwendigkeit häufiger endotrachealer Absaugung und lebensbedrohliche Hypoxämie mit Sauerstoffsättigung $<85 \%$ trotz Sauerstoffzufuhr über Gesichtsmaske.

\section{Beatmungszugang}

Zum Einsatz kommen Nasenmasken [4, $16,19,39,48]$ oder nasoorale Gesichtsmasken (Vollgesichtsmasken; [9, 18, 36, 53, 55]). Vorteile von Nasenmasken sind 
Tabelle 3

Mögliche Beatmungsmodi der nicht-invasiven Beatmung

\begin{tabular}{|c|c|}
\hline Indikation & Beatmungsmodus \\
\hline Hypoxie mit Normo- oder Hypokapnie (z. B. Lungenödem) & Nasale CPAP-Atmung (nCPAP, keine Beatmung) \\
\hline Hyperkapnie (z. B. COPD, Kyphoskoliose, neuromuskuläre Erkrankungen) & $\begin{array}{l}\text { CPAP/ASB (BiPAP }{ }^{\oplus} \text {, BiLevel-CPAP o.ä.) oder } \\
\text { Assistiert kontrollierte Beatmung (druck- oder volumenkontrolliert } \\
\text { je nach Gerät) }\end{array}$ \\
\hline
\end{tabular}

CPAP:Spontanatmung mit kontinuierlichem positiven Atemwegsdruck, ASB: assistant spontaneous breathing.

meist bequemerer Sitz, weniger klaustrophobische Reaktionen des Patienten, kleinere Totraumvolumina und die Möglichkeit zu husten oder zu sprechen.

Patienten mit schwerer Dyspnoe atmen meist durch den Mund. Deshalb haben Nasenmasken eine größere Leckage und damit geringere Effektivität. Diese Nachteile treten bei Vollgesichtsmasken weniger auf. Diese sind jedoch unbequemer, führen zu mehr klaustrophobischen Reaktionen, müssen zum Husten und Sprechen abgenommen werden und bei Erbrechen besteht Aspirationsgefahr. Außerdem ist der Totraum größer. Klinisch ist dies jedoch kaum relevant.

\section{Beatmungsmodus}

Bei Hypoxie mit Normo- oder sogar Hypokapnie, wie man sie z. B. beim Lungenödem findet, ist die nasale CPAPAtmung („,continous positive airway pressure", Spontanatmung mit PEEP, keine Beatmung) mit dem Ziel der Erhöhung der funktionellen Vitalkapazität indiziert (Tabelle 3). Ein nCPAP wird i.d.R. gut toleriert, erhöht allerdings die exspiratorische Atemarbeit. Beim hyperkapnischen Atemversagen ist kein positiver Effekt zu erwarten.

Bei Hyperkapnie ist eine alleinige nCPAP-Therapie nicht ausreichend, da die Atemmuskulatur nicht entlastet wird. Hier müssen assistierende Verfahren (CPAP/ASB, BiPAP ${ }^{\circledast}$, BiLevel-CPAP o. ä., druck- oder volumenkontrollierte assistierte Beatmung) in der Einleitungsphase eingesetzt werden, da sie besser als eine kontrollierte Beatmung (CMV) toleriert werden. Assistierte Beatmungsformen führen nur zur Teilentlastung der Atemmuskulatur. Synchronisierung von Patient und Maschine ist jedoch deutlich leichter und schneller zu erreichen als unter CMV.
Bei langfristig durchgeführter intermittierender Selbstbeatmung ist die kontrollierte Beatmung vorteilhaft. Nur so wird während der Beatmungszeit die Atemmuskulatur komplett entlastet und ein ausreichender Erholungseffekt erzielt. Voraussetzung ist, dass der Patient seine eigene Atemaktivität völlig einstellt.

Die druckkontrollierte Beatmung (PSV) wird i.d.R. besser toleriert als volumenkontrollierte und wird in den meisten Studien als Methode der Wahl angegeben $[9,18,36,55]$. Ein PEEP verbessert meist die Oxigenierung. Allerdings steigt der für eine ausreichende Ventilation nötige Atemwegsdruck und damit der Maskendruck (erhöhte Nebenwirkungsrate, insbesondere Leckage). Ein PEEP sollte daher individuell und möglichst niedrig eingesetzt werden.

\section{Beatmungsgeräte}

Grundsätzlich kann NIV mittels Maske auch mit den konventionellen Beatmungsgeräten (Evita IV, Bennet 7200 u. a.) durchgeführt werden. Das großlumige Schlauchsystem mit inspiratorischem und exspiratorischem Schenkel ist aber ungünstig (Gewicht an der Maske, Reduktion der Bewegungsmöglichkeit, großer Totraum). Die Konstruktion eigener „Systeme“ aus Einmalschläuchen ist rechtlich schwierig. Die Einmalmaterialien müssen ein „CE-Zeichen“ haben und müssen vom Gerätehersteller als Zubehör zugelassen sein (Medizinproduktegesetz!). Die Trigger-Einrichtungen sind teilweise zu unempfindlich. Deshalb treten häufig Fehlalarme auf, besonders „Low-pressure“- oder „Low-volumeAlarme" bei Leckagen.

Spezielle elektrisch angetriebene Beatmungsgeräte mit leichten Einschlauchsystemen, die für die häusliche
Beatmung entwickelt wurden, sind für die Maskenbeatmung zu bevorzugen. Problem bei den Geräten ohne Exspirationsschlauch ist das Fehlen eines Monitorings der Beatmungsparameter (Atemminutenvolum, Atemzugvolumen u. a.). Das Kohlendioxid wird durch ein Loch (Ausatemöffnung) in der Maske, ein Schlitzventil oder ein maskennah angebrachtes pneumatisch gesteuertes Ventil abgeatmet.

\section{Gerätetypen}

Prinzipiell lassen sich 3 Gerätetypen unterscheiden. Nasale CPAP- (nCPAP-) Geräte aus der Therapie des obstruktiven Schlafapnoesyndroms, die einen kontinuierlichen Inspirationsgasfluss erzeugen, können eingesetzt werden, wenn die Anwendung von CPAP ohne, ,echte“ Beatmung ausreichend ist. Vorteil ist die sehr einfache Anwendung und die gute Akzeptanz durch die Patienten, zumindest bei mäßigen Druckwerten $<12$ mbar.

Ventillose Beatmungsgeräte - sog. Bilevel- oder BiPAP-Geräte - sind meist Weiterentwicklungen der nasalen CPAPGeräte aus der Therapie des obstruktiven Schlafapnoesyndroms (z. B. Respironics BiPAP ST/T). Diese Geräte werden vor allem in Kliniken eingesetzt, die Erfahrung in der Heimbeatmung haben und die Geräte auch im Intensivbereich verwenden. Bilevel oder BiPAP - mit kleinem „i“ - entspricht nicht dem BIPAP - mit großem „I“ - der großen Beatmungsgeräte auf der Intensivstationen wie z. B. der Evita 4 von Dräger.

Typischer Beatmungsmodus dieser Geräte ist die druckunterstützte Spontanatmung (Pressure Support Ventilation, PSV). Bei einigen Geräten ist zusätzlich durch eine Zeitsteuerung eine druckkontrollierte Beatmung (Pressure Control Ventilation, PCV) möglich. Der 
inspiratorische (IPAP) und exspiratorische $($ EPAP $=$ PEEP) Druck wird durch Drehzahländerung der Antriebsturbine erzeugt. Die mit $\mathrm{CO}_{2}$ angereicherte $\mathrm{Ex}$ spirationsluft wird durch den kontinuierlichen Frischgasfluss aus in der Maske oder maskennah angebrachten kleinen Öffnungen ausgeblasen. In einer Untersuchung am Lungenmodell waren die meisten der getesteten Bilevel-Geräte bezüglich der Leistungsfähigkeit bei hohem ventilatorischem Bedarf einem konventionellen Beatmungsgerät (Puritan-Bennet 7200) gleichwertig oder sogar überlegen [10]. Allerdings gibt es Berichte über die Gefahr einer $\mathrm{CO}_{2}$-Rückatmung bei der BiPAP-Therapie [17]

Ventilgesteuerte Geräte mit einem patientennah im Schlauch angebrachten und pneumatisch gesteuerten Exspirationsventil gibt es als volumengesteuerte (z. B. Life-care PLV 100, Breas PV 501) oder druckkontrollierten (PCV) sowie druckunterstützte (PSV; z. B. Breas PV 401) Geräte. Ein PEEP lässt sich meist nur relativ ungenau durch ein auf das Ausatemventil aufgesetztes mechanisches PEEP-Ventil einstellen.

\section{Weitere Funktionen}

Die zusätzliche Anreicherung der Inspirationsluft mit Sauerstoff ist nicht direkt an den Geräten einstellbar. Die $\mathrm{O}_{2}$ - Zumischung erfolgt „nach Gefühl“ direkt in Maske oder Schlauchadapter. Dabei hängt der $\mathrm{F}_{\mathrm{i}} \mathrm{O}_{2}$ nicht nur von der eingestellten $\mathrm{O}_{2}$-Flussrate ab. Er ändert sich auch in Abhängigkeit vom Flow des Beatmungsgerätes. So nimmt z. B. der $\mathrm{F}_{\mathrm{i}} \mathrm{O}_{2}$ durch eine kompensatorische Flusssteigerung bei einer Leckage $a b$, ohne das der $\mathrm{F}_{\mathrm{i}} \mathrm{O}_{2}$-Abfall angezeigt oder über einen Alarm erkannt wird. Im Gegensatz zu den konventionellen Beatmungsmaschinen ist eine definierte inspiratorische $\mathrm{O}_{2}$-Konzentration bei den meisten $\mathrm{Ge}$ räten nicht einstellbar.

Bei den NIV-Maschinen sind die Alarmfunktionen des exspiratorischen Volumenmonitorings der konventionellen Beatmungsgeräte wie „low volume“, „Volumen inkonstant“ u. a. nur eingeschränkt vorhanden. Einige Geräte können als Sonderversionen für den stationären Einsatz mit zusätzlichen Monitoring- und Alarmeinrichtungen ausgestattet werden. Grundsätzlich sind die Geräte gegenüber großen Beatmungsgeräten leichter und unkomplizierter bedienbar.
Als Masken sind kommerzielle Nasenmasken aus Silikon-Kunststoff in verschiedenen Größen verfügbar. Die Befestigung erfolgt mit Haltebändern. Wichtig ist, dass die Masken für eine nCPAP-Therapie teilweise eine Ausatemöffnung für die $\mathrm{CO}_{2}$-Abatmung haben. Bei Geräten mit kontinuierlichem Fluss muss die Ausatemöffnung offen sein, bei Geräten mit Exspirationsventil aber zugeklebt werden. Bei der „Veränderung“ der Maske muss an die Probleme mit dem Medizinproduktegesetz gedacht werden. Optimal ist die Verfügbarkeit von entsprechenden Masken für die jeweiligen Gerätetypen. Mund-Nasen-Masken (Gesichtsmasken) sollten wegen der erhöhten Aspirationsgefahr nur ausnahmsweise gebraucht werden!

Bei den Beatmungsgeräten für die Maskenbeatmung muss zwischen Gerät und Inspirationsschlauch ein Beatmungsfilter gesetzt werden. Der Filter ist ein Schutz vor einer Kontamination des Beatmungsgerätes.

\section{Praktische Durchführung der NIV}

Bei der praktischen Durchführung auf der Station haben sich bei uns entsprechende Kurzanweisungen (Abb. 1) bewährt, auf denen Geräteaufbau und Grundeinstellung kurz zusammengefasst sind.

Tabelle 4 gibt einen Überblick über die praktische Durchführung der NIV auf der Intensivstation. Nach Information des Patienten, Wahl von Gerät und passender Maske - je nach Gerät muss die Ausatemöffnung verschlossen sein oder darf nicht verschlossen werden wird die $\mathrm{O}_{2}$-Zumischung entsprechend der aktuellen pernasalen/peroralen $\mathrm{O}_{2}$ Gabe eingestellt. Die Maske muss anfangs von Hand vorgehalten und Patient sowie $\mathrm{O}_{2}$-Sättigung beobachtet werden. Besonders bei somnolenten Patienten mit $\mathrm{CO}_{2}$-Narkose ist initial ein manuelles Abdichten des Mundes notwendig. Erst bei ausreichender Toleranz - nach Adaptation und Synchronisation - darf die Maske mit Bändern festgeschnallt werden. Entscheidend für die Akzeptanz einer NIV ist das Wohlbefinden des Patienten! In diesem banalen Satz liegt das Geheimnis von Erfolg oder Misserfolg einer nicht-invasiven Beatmung.

\section{Grundeinstellungen}

Initiale Einstellung und Anpassung der Geräte sind abhängig von der Indikation. Beim Lungenödem wird zunächst eine druckunterstützte Spontanatmung nCPAP - mit relativ niedrigem Druck ca. $10 \mathrm{~cm} \mathrm{H}_{2} \mathrm{O}$ - durchgeführt. Der Beatmungsdruck wird langsam bis auf maximal $25 \mathrm{~cm} \mathrm{H}_{2} \mathrm{O}$ gesteigert, bis eine ausreichende Oxigenierung $\left(\mathrm{S}_{\mathrm{a}} \mathrm{O}_{2}\right.$ !) erreicht ist. In der letzten Zeit wird bei Patienten neben der nCPAP-Therapie eine druckgesteuerte Beatmung diskutiert, um auch bei diesen Patienten die Atemmuskulatur zu entlasten.

Bei Patienten mit hyperkapnischem Lungenversagen - z. B. exazerbierte COPD-Patienten ist das Ziel die kontrollierte Beatmung. Bei zunächst vom Personal gehaltener Maske wird ein niedriger Beatmungsdruck von z. B. $10 \mathrm{~cm} \mathrm{H}_{2} \mathrm{O}$ bzw. ein niedriges Zugvolumen von 3-5 $\mathrm{ml} / \mathrm{kg}$ eingestellt. Als Maschinenfrequenz sollten zunächst 2 Atemzüge über der Atemfrequenz des Patienten gewählt werden. Sinnvoll ist das aber nur, solange die Atemfrequenz des Patienten $<30 / \mathrm{min}$ liegt.

Beatmungsdruck - bis maximal $25 \mathrm{~cm} \mathrm{H}_{2} \mathrm{O}$ - bzw. Zugvolumen werden langsam gesteigert, bis eine ausreichende Ventilation erreicht ist, der $\mathrm{pH}$-Wert steigt bzw. $\mathrm{P}_{\mathrm{a}} \mathrm{CO}_{2}$ abfällt. Die maschinelle Frequenz wird stufenweise auf 10-14 Atemzüge reduziert. Die Beatmungsparameter - $\mathrm{P}_{\text {insp }}$, insp. Flow, Plateau, I:EVerhältnis - müssen individuell angepasst werden und richten sich nach der Tolerierung durch den Patienten. Häufiges Problem bei obstruktiven Patienten ist die Entstehung eines Auto-PEEP durch "trapped air" infolge zu hoher Atemfrequenz und/oder zu langem I:EVerhältnis.

Da den NIV-Geräten die üblichen Alarme der konventionellen Beatmungsgeräte fehlen, ist die engmaschige Betreuung und Überwachung durch Ärzte sowie Pflegepersonal zwingend erforderlich. Neben der Veränderung von Resistance und Compliance beim Patienten kann es durch Verrutschen der Maske oder Mundöffnung (bei nasaler Maske) plötzlich zur Hypoventilation kommen, ohne dass ein Maschinenalarm gegeben wird. Grundsätzlich kann im Gegensatz zur konventionellen Beatmung bei NIV nie von konstanten Beatmungsparametern (AZV, PEEP etc.) ausgegangen wer- 



\section{Übersicht}

\section{BREAS PV 401 / Bedienungsanleitung}

Firma: BREAS

Vertrieb: METEC

Cave:

- o Akku, nur Netzbetrieb

- wenn PEEP erwünscht, muß ein PEEP.Ventil auf das Ausatemventil gesetzt werden

- nach Nahrungsaufnahme ca. 30 - 60 Minuten Pause vor Beginn der Beatmung

\section{Autbau:}

- Gerát $\rightarrow$ Filter $\rightarrow$ BAEAS - Schlauch, weiBer Schlauch fùr Druckmessung und grûner Steuerschlauch fûr Ausatermventil $\rightarrow \mathrm{O}_{2}$-AnschluB (über T-Stück im Schlauchsystem oder direkt in Maske), Maske ohne Ausatemöffnung $\rightarrow$ Kopt/Kinnband

\begin{tabular}{|c|c|c|}
\hline & & Pat. in $\mathrm{CO}_{z}$-Narkose / resp. Erschöpfung \\
\hline Modusauswahl: & & PCV \\
\hline Druck & (mber) & 12 , stufernweise steigern bis max 25 \\
\hline Atemfrequenz & $\left.(\min )^{\prime}\right)$ & $\begin{array}{l}\text { Beginn mit } 2 \text { AF uber der Eigen-AF (nur sinnvoll } \\
\text { wenn Eigen-AF }<30 / \text { min). } \\
\text { dann stufenweise reduzieren bis auf } 10-14 / \text { min }\end{array}$ \\
\hline Plateau & & 9 (dimensionsios) \\
\hline Inspirationszeit & & $\begin{array}{l}\qquad 1,2-0,9 \mathrm{~s} \\
\text { (nach gewünschtem I:E-Verhälttnis (Errechnen I)) }\end{array}$ \\
\hline Besonderheiten & & $\begin{array}{l}\text { Theophyllin-Pertusor } \\
\text { Atosil / Morphin (Toleranz der Maske) } \\
\text { Paspertin }\end{array}$ \\
\hline
\end{tabular}

\section{Quantum ${ }^{\oplus}$ PSV / Bedienungsanleitung}

Firma: Healthdyne

Vertrieb: Heinen und Löwenstein

Cave:

- o Akku, nur Netzbetrieb möglich

- nach Nahrungsautnahme ca. 30 - 60 Minuten Pause vor Beginn der Beatmung

Aufbau

- Gerät $\rightarrow$ Filter $\rightarrow \mathrm{O}_{2}$-Anschlu $\mathrm{B} \rightarrow$ Schlauch $\rightarrow$ Maske mit Ausatemöffnung z.B. vo Healthdyne $\rightarrow$ Kopt- und Kinnband

\begin{tabular}{|c|c|c|c|}
\hline & & $\begin{array}{c}\text { Pat. im } \\
\text { Lungenödem }\end{array}$ & $\begin{array}{l}\text { Pat. in } \mathrm{CO}_{2} \text {-Narkose / } \\
\text { resp. Erschopfung }\end{array}$ \\
\hline Modusauswahl: & & CPAP & Spontan/Zeit \\
\hline \multicolumn{4}{|l|}{ Patienteneinstellung } \\
\hline CPAP / EPAP & $\left(\mathrm{om} \mathrm{H} \mathrm{H}_{-} \mathrm{O}\right)$ & $\begin{array}{l}3-8 \\
\text { (bei Pat. mit } \\
\text { schwerer COPD } \\
\text { max. 5) }\end{array}$ & 3 \\
\hline IPAP & $\left(\mathrm{Om} \mathrm{H}_{2} \mathrm{O}\right)$ & $\begin{array}{l}\text { nicht zwingend, } \\
\text { initial } 3.5 \mathrm{~cm} \mathrm{H}_{2} \mathrm{O} \\
\text { über EPAP móglich }\end{array}$ & $\begin{array}{l}12 \text {, stufenweise steigern } \\
\text { bis max } 25\end{array}$ \\
\hline Atemfrequenz & ( $\min )$ & entiâlit & $\begin{array}{l}\text { Beginn mit } 2 \text { AF über der } \\
\text { Eigen-AF (nur sinnvoll } \\
\text { wenn Eigen-AF }<30 / \text { min), } \\
\text { dann stufernweise } \\
\text { reduzieren bis } \\
\text { aut } 10.14 / \mathrm{min}\end{array}$ \\
\hline Anstiegszeit & & entiâllt & $0,9 \mathrm{sec}$ \\
\hline Inspirationszeit & & enttállt & $33 \%$ \\
\hline Besonderheiten & & 0 & $\begin{array}{l}\text { Theophyllin-Perfusor } \\
\text { Atosil / Morphin (Toleranz } \\
\quad \text { der Maske) } \\
\text { Paspertin }\end{array}$ \\
\hline
\end{tabular}

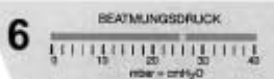

- ofuck fe at at ma

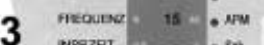

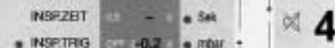

patew $\pi \quad h$.

Expsts $=30=$.

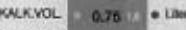

SAweins:

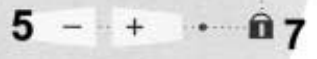

DANAB

- sencalun

inuo

2

-1 moat

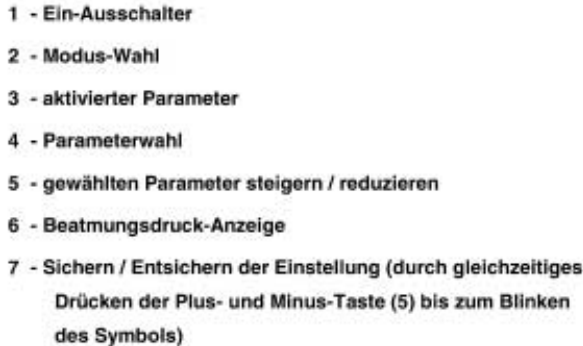

des Symbols)
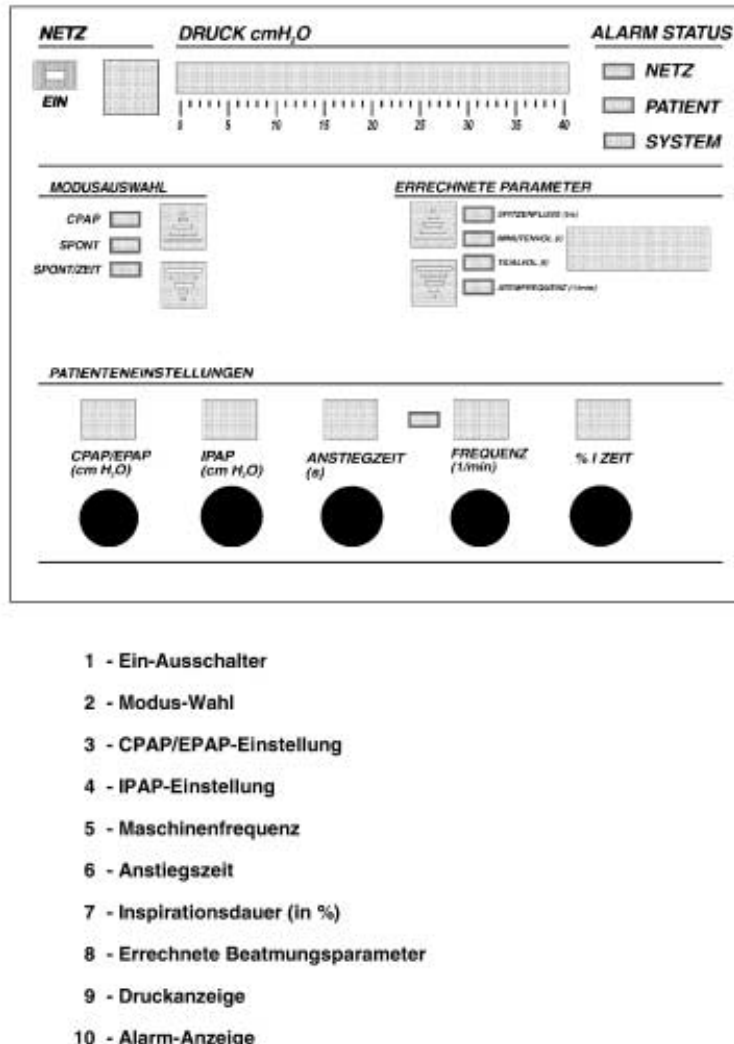
Tabelle 4

Praktische Durchführung der nicht-invasiven Beatmung

Information des Patienten

Geräteaufbau kontrollieren:

- Beatmungsfilter?

- Ausatemöffnung: offen (bei Systemen ohne Ausatemventil) oder verschlossen

(bei Systemen mit Ausatemventil)

- Maske (richtige Größe?)

$\mathrm{O}_{2}$-Zufuhr entsprechend der aktuellen pernasalen/peroralen $\mathrm{O}_{2}$-Gabe einstellen

Grundeinstellung überprüfen:

- Druckunterstützte Spontanatmung

- Relativ niedriger Druck: ca. $10 \mathrm{~cm} \mathrm{H}_{2} \mathrm{O}$

- Niedriges Atemzugvolumen $3-5 \mathrm{ml} / \mathrm{kg} \mathrm{KG}$

- Frequenz: 2 Atemzüge über Eigenfrequenz (wenn diese $<30 / \mathrm{min}$ )

- Beatmungsparameter ( $P_{\text {insp }}$, Flow, evtl. Frequenz, Plateau, I:E-Verhältnis) individuell anpassen

Maske anfangs mit der Hand halten

Bei Nasenmasken Patienten zum konsequenten Schließen des Mundes anhalten

Besonders bei somnolenten Patienten ( $\mathrm{CO}_{2}$-Narkose) initial manuelles Abdichten des Mundes

Patient und $\mathrm{O}_{2}$-Sättigung beobachten.

Erst bei ausreichender Toleranz Maske mit Bändern befestigen

Entscheidend für den Erfolg ist das Wohlbefinden des Patienten!

\section{Anwendungsgebiete}

Erste Berichte über den Einsatz einer „Überdruck-Atmung“ mittels Maske beim Asthma bronchiale [2] und beim Lungenödem [3] stammen schon aus den 1930er Jahren. Aber erst Ende der 1970er Jahre wurden der Einsatz von CPAP mittels Maske beim hypoxämischen Lungenversagen $[22,50]$ und Ende der 1980er Jahre der Einsatz einer NIV mit intermittierendem positivem Atemwegsdruck (IPPV) mittels Maske beim hyperkapnischen Lungenversagen $[8$, 34] in unkontrollierten Studien untersucht (Übersicht bei [32]). Prospektiv randomisierte Daten - überwiegend bei COPD-Patienten, wurden erst Mitte der 1990er Jahre publiziert. Die größten Erfahrungen bestehen zur Zeit mit der NIV bei COPD-Patienten.

Für die Indikation zur NIV wird zwischen einem hyperkapnischen ventilatorischen Versagen infolge eingeschränkter alveolärer Ventilation (respiratorischer Globalinsuffizienz) - z. B. bei COPD und einem hypoxämischen Lungenversagen durch Gasaustauschstörung (respiratorische Partialinsuffizienz) - z. B. beim Lungenödem - unterschieden. Während beim hyperkapnischen Versagen primär eine NIV mit intermittierendem positivem Atemwegsdruck (IPPV) indiziert ist, wird bisher beim hypoxämischen Versagen die Anwendung eines kontinuierlichen positiven Atemwegsdrucks (CPAP)

für ausreichend angesehen.

reicht wird. Abbruchkriterien sind neben den Kontraindikationen eine Verschlechterung von Klinik, Ventilation und Gasaustausch sowie fehlende Toleranz durch den Patienten.

\section{Monitoring}

Beim Monitoring einer NIV (Abb. 2 ) ist zunächst die subjektive Einschätzung des Patienten von Bedeutung. Hat der Patient das Gefühl, die Beatmung hilft oder hilft nicht? Hinweise geben objektivierbare Parameter wie Atemfrequenz, Atemrhythmus und Einsatz der Atemmuskulatur (paradoxe Bewegung des Abdomens?). Weiter muss auf Leckagen an der Maske, Druckstellen durch die Maske, bei Nasenmasken auf Mund-

Abb. 1a-d \ Hinweisblatt am Beatmungsgerät (entwickelt von M. Klocke und E. Meissner; eingesetzt auf einer internistischen Intensivstation der MHH; jeweils Vorder- und Rückseite)

schluss sowie den korrekten Sitz der Haltebänder geachtet werden. Die Einstellung des Beatmungsgerätes (AF, AZV Beatmungsdruck, Inspirationszeit, u. a.) muss im Zusammenhang mit den Atembemühungen des Patienten überprüft und angepasst werden.

Technische Parameter wie Herzfrequenz, Blutdruck und $\mathrm{O}_{2}$-Sättigung sowie die Blutgasanalyse $\left(\mathrm{pH}, \mathrm{P}_{\mathrm{a}} \mathrm{O}_{2}, \mathrm{P}_{\mathrm{a}} \mathrm{CO}_{2}\right)$ erlauben die Effektivität der NIV abzusichern (Tabelle 5).

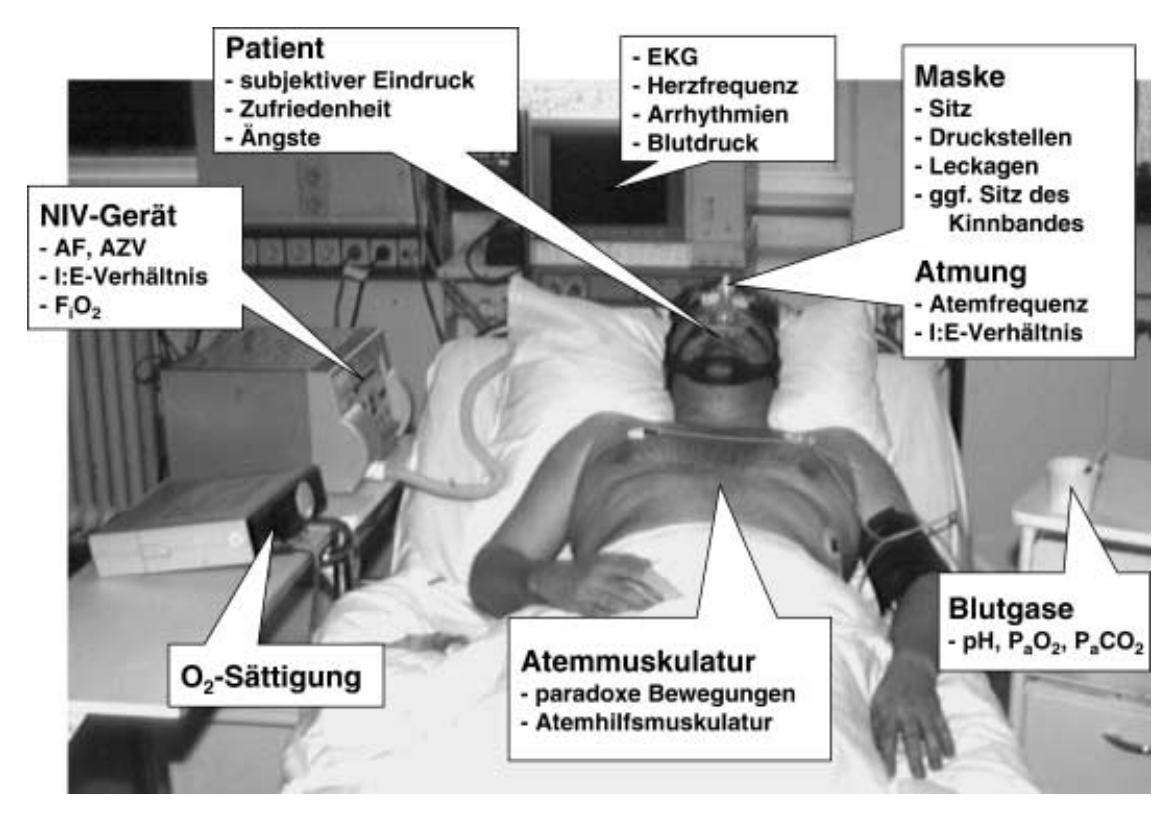

Abb. $2 \Delta$ Monitoring einer nicht-invasiven Beatmung 

Tabelle 5

Erfolgsparameter und Abbruchkriterien der nicht-invasiven Beatmung

\begin{tabular}{lll} 
Parameter & Erfolgskriterien & Abbruchkriterien \\
\hline Klinik & Sichtbare Entlastung der Atempumpe $(\mathrm{AF} \downarrow, \mathrm{HF} \downarrow)$ & Unruhe $\uparrow$, Gegenwehr $\uparrow$, Luftnot $\uparrow$ \\
& Beruhigung/Entspannung des Patienten & Progrediente Bewusstseinstrübung \\
Alveoläre Ventilation & Gesteigert $\left(\mathrm{P}_{\mathrm{a}} \mathrm{CO}_{2} \downarrow\right)$ & Hyperkapnie $\uparrow$ trotz Beatmung \\
Oxigenierung & Besser $\left(\mathrm{S}_{\mathrm{a}} \mathrm{O}_{2}>90 \%\right)$ & Fortbestehende Hypoxie $\left(\mathrm{S}_{\mathrm{a}} \mathrm{O}_{2}<85 \%\right)$ \\
Probleme & & Bedrohliche Arrhythmien oder Kreislaufinstabilität \\
& & Nicht beherrschbare Maskenprobleme
\end{tabular}

In einer Metaanalyse ließ sich bei Patienten mit schwerer akuter respiratorischer Insuffizienz nur für die Gruppe der COPD-Patienten eine signifikante Reduktion der Notwendigkeit zur Intubation sowie der Mortalität zeigen [26]. Für die akute respiratorische Insuffizienz bei „Nicht-COPD-Patienten“ ließ sich in der Metaanalyse kein signifikanter Vorteil nachweisen [26].

\section{Kardiale Insuffizienz (Lungenödem)}

Zum Einsatz der NIV beim kardial bedingten Lungenödem liegen eine Reihe von Studien vor, wobei sowohl nur CPAP $[5,30,44]$ als auch IPPV mit und ohne PEEP $[1,25,37,42,45]$ zur Anwendung kamen. Wegen der einfacheren Technik wurde meist CPAP eingesetzt. In einer Metaanalyse konnte durch die nCPAP-Therapie die Notwendigkeit einer Intubation um 26\% und die Mortalität im Krankenhaus um 6,6\% reduziert werden.

In letzter Zeit wird der routinemäBige Einsatz der IPPV zur Entlastung der Atemmuskulatur diskutiert. Erste Ergebnisse beim Vergleich der Effektivität von IPPV im BIPAP-Modus vs. CPAP zeigen signifikant schnellere Besserungen unter IPPV [37]. Allerdings waren die Anzahl der Myokardinfarkte in der IPPV-Gruppe (71\%) größer als in der CPAP-Gruppe $(31 \%, \mathrm{p}<0,06)$ und andere Studienendpunkte (Beatmungsdauer, Aufenthalt auf Intensivstation und im Krankenhaus, Intubationsrate, Mortalität) zwischen den Gruppen nicht unterschiedlich. Hinweise auf eine möglicherweise erhöhte Rate an kardialen Komplikationen unter NIV fanden sich in einer weiteren Arbeit [45]. Als beeinflussender Faktor ist der Stress zu diskutieren, den die Einlei- tung einer NIV für die Patienten bedeuten kann. Zur Zeit darf deshalb die NIV bei Patienten mit schwerer Koronarinsuffizienz bzw. frischem Herzinfarkt nicht eingesetzt werden.

\section{Chronisch-obstruktive Lungenerkrankung}

Für Patienten mit COPD und einem hyperkapnischen Versagen wurde zu Beginn der 1990er Jahre nach einer Dauer von 45 min CPAP mittels Maske ein signifikanter Anstieg von $\mathrm{P}_{\mathrm{a}} \mathrm{O}_{2}$ und $\mathrm{pH}$ sowie ein signifikanter Abfall von $\mathrm{P}_{\mathrm{a}} \mathrm{CO}_{2}$ und Atemfrequenz gezeigt [8]. Verglichen mit einem historischen Kollektiv unter Intubation und konventioneller Beatmung waren unter CPAP die Beatmungsdauer und Dauer der Behandlung auf der Intensivstation signifikant kürzer.

Die Verbesserung der Blutgase unter NIV bei Exazerbation einer COPD wird durch eine gesteigerte alveoläre Ventilation und nicht durch Verbesserung des Ventilations-Perfusions-Verhältnisses hervorgerufen [15].

In prospektiv randomisierten Studien zum Vergleich einer alleinigen Standardtherapie vs. Standardtherapie mit NIV konnte für die Gruppe, die zusätzlich NIV bekam, eine signifikante Reduktion von endotrachealen Intubationen, Komplikationen, Krankenhaustagen und Mortalität gezeigt werden (CPAP: [9], IPPV: [6]).

Nur eine prospektiv randomisierte Studie zeigte für das Gesamtkollektiv keinen Vorteil für die zusätzliche Anwendung einer NIV bei COPD-Exazerbationen [55]. In der Arbeit ergaben sich nur bei den Patienten, die einen erhöhten $\mathrm{P}_{\mathrm{a}} \mathrm{CO}_{2}$ (>45 mmHg) aufwiesen, Vorteile für die NIV. Als weiterer Vorteil ergab sich für NIV gegenüber konventioneller Beatmung eine Reduktion von personellem und finanziellem Aufwand [38].

\section{Asthma bronchiale}

Über die Anwendung der NIV beim schweren Asthmaanfall gibt es sowohl für den Einsatz von CPAP [49] als auch IPPV [4, 35, 42] Berichte. Kontrollierte Studien fehlen allerdings bisher. Der Versuch einer NIV - vorzugsweise IPPV + PEEP - erscheint gerechtfertigt.

\section{Cystische Fibrose}

Zum Einsatz der NIV bei cystischer Fibrose liegen nur unkontrollierte Studien vor [24, 41]. Die Maskenbeatmung in Akutsituationen ist nach eigener Erfahrung wegen Sekretproblem schwierig. Ein Versuch mit NIV erscheint insbesondere bei Patienten, die zur Lungentransplantation vorgesehen sind, als „bridging" möglich [24].

\section{Lungenfibrose}

Zum Einsatz der NIV bei Lungenfibrosen liegen keine kontrollierten Daten vor. Nach eigener Erfahrung ist durch CPAP im Einzelfall eine verbesserte Oxigenierung erreichbar. Eine IPPV-Beatmung ist wegen notwendiger hoher Beatmungsdrücke und Atemfrequenz nicht erfolgversprechend.

\section{Pneumonie}

Bei ambulant erworbenen Pneumonien wurden sowohl für CPAP $[7,51]$ als auch für IPPV $[11,36,54]$ Studien mit kleinen Fallzahlen und widersprüchlichen Ergebnissen publiziert. In der bisher einzigen prospektiv randomisierten Studie, 
in der bei Patienten mit ambulant erworbenen Pneumonie die Standardtherapie vs. NIV mit Standardtherapie untersucht wurde, ließ sich eine Reduktion der Intubationsrate (von $50 \%$ auf $21 \%$; $\mathrm{p}=0,03)$ und der Zeit auf der Intensivstation (von $6,0 \pm 1,8$ auf $1,8 \pm 0,7$ Tagen; $\mathrm{p}=0,04)$ durch die zusätzliche NIV-Therapie nachweisen. Die gesamte Zahl der Krankenhaustage und die Mortalität unterschieden sich zwischen den beiden Gruppen aber nicht [12]. Allerdings kann in der Studie eine Beeinflussung durch die COPD-Patienten (12/28 Patienten in der NIV-Gruppe, 11/28 Patienten in der Gruppe mit alleiniger Standardtherapie) nicht völlig ausgeschlossen werden. Ein Therapieversuch mit nicht-invasiver Beatmung ist gerechtfertigt, sollte aber die Intubation und konventionelle Beatmung nicht wesentlich verzögern.

Die respiratorische Insuffizienz bei Pneumocystis-carinii-Pneumonie infolge AIDS weist bei Intubation und Beatmung eine relativ schlechte Prognose auf. Um die Intubation $\mathrm{zu}$ vermeiden und möglicherweise dadurch die Prognose zu verbessern, kann nach verschiedenen publizierten Studien auch CPAP $[14,20,23]$ oder IPPV $[34,36,43]$ sinnvoll eingesetzt werden. In den - allerdings unkontrollierten Studien - hatten Patienten, die mit CPAP oder IPPV therapiert werden konnten, eine geringere Mortalität als die Patienten, die intubiert werden mussten $[20,23]$.

\section{Acute respiratory distress syndrome}

Beim ARDS wurde in Einzelberichten ein günstiger Effekt von CPAP [13, 47] sowie IPPV [34, 36, 40, 54] beschrieben. Größere und kontrollierte Studien fehlen bisher. Bei leichteren Formen ist initial ein Therapieversuch möglich. Das ARDS mit ausgeprägter Hypoxie, wenn z. B. eine verlängerte Inspirationszeit nötig ist, wird aber der Intubation und konventionellen Beatmung vorbehalten bleiben.

\section{Weaning}

Für Patienten mit Problemen im Weaning nach Beatmung ist die NIV eine neue Option [29, 31, 46]. Sowohl für COPD-Patienten [21] wie auch andere Patienten [21, 27] konnten in kontrollierten Studien positive Effekte nachgewiesen werden. Die Dauer der endotrachea- len Intubation war in der NIV-Gruppe signifikant geringer als in der Pressuresupport-Gruppe. Gleichzeitig war aber die gesamte Dauer der Beatmungsunterstützung in der PSV-Gruppe signifikant geringer als in der NIV-Gruppe. Die Erfolgsrate des Weaning war zwischen den Gruppen nicht unterschiedlich [21].

Ein Einsatz der Maskenbeatmung zum Weaning von intubierten Patienten kann grundsätzlich empfohlen werden und im Einzelfall die Intubationsdauer deutlich verkürzen. Ein technisches Problem ergibt sich durch die heute bei längerfristigen Beatmungen häufig durchgeführte Tracheotomie. Der Übergang vom Tracheostoma mit konventioneller Beatmung auf NIV ist schwierig. Nach dem operativen Verschluss eines plastischen Tracheostomas darf für 10-14 Tage keine NIV durchgeführt werden. Die Druckbelastung infolge der Beatmung mit positivem inspiratorischem Atemwegsdruck und evtl. PEEP würde sonst zu Wundheilungsstörungen und Nahtinsuffizienz führen.

Die Punktionstracheotomie bietet hier Vorteile, da sich die Öffnung nach Entfernung der Kanüle meist innerhalb weniger Tage durch Schrumpfung verschließt. Eine NIV ist in dieser Zeit nur schwierig möglich. Ein alleiniges „Abkleben“ der Öffnung hält dem positivem inspiratorischem Atemwegsdruck nicht stand. In den ersten Tagen muss i.d.R. zusätzlich manuell komprimiert werden. Dadurch wird die Anwendung auf wenige Stunden am Tag begrenzt. In die Indikationsstellung für eine Tracheostomie sollte die Überlegung zum Vorgehen im Weaning eingehen.

\section{Rettungsdienst}

Im Rettungsdienst hat sich bisher die NIV als Alternative zur Intubation mit konventioneller Beatmung nicht durchgesetzt, obwohl die technischen Voraussetzungen - mittels Akku über 6-8 h netzunabhängig zu betreibende Geräte gegeben sind und der sinnvolle Einsatz für Lungenödem, Asthma bronchiale und chronisch obstruktive Lungenerkrankung belegt ist.

\section{Zusammenfassung}

Bei akuter respiratorischer Insuffizienz ist häufig eine mechanische Beatmung notwendig. Diese wird meist mittels en- dotrachealer Intubation durchgeführt, die jedoch sowohl akut als auch chronisch mit Problemen und Komplikationen belastet ist. Eine Alternative bietet hier die nicht-invasive Beatmung (NIV) mittels Maske. Diese hat sich allerdings aus verschiedenen Gründen (apparative Voraussetzungen, mangelnde Erfahrung des Personals) auf den Intensivstationen noch nicht etabliert.

Neben dem Erhalt physiologischer Bedingungen ist der verminderte Medikamentenbedarf ein weiterer Vorteil der NIV, nachteilig ist vor allem in der Eingewöhnungsphase der erhöhte personelle Aufwand. Indiziert ist die NIV prinzipiell nur bei wachen, kooperativen Patienten mit akuter respiratorischer Insuffizienz, wenn eine Beatmungsdauer unter $48 \mathrm{~h}$ erwartet wird.

Verschiedene Beatmungsmasken und -geräte erlauben verschiedene Beatmungsmodi, die individuell entsprechend der Indikation eingesetzt werden. Klinische Studien belegen für die chronisch-obstruktive Lungenerkrankung Vorteile der Maskenbeatmung. Bei anderen Indikationen erlauben die verfügbaren Daten noch kein gesichertes Urteil, rechtfertigen aber einen Behandlungsversuch.

Bei der praktischen Durchführung haben sich entsprechende Kurzanweisungen bewährt, auf denen Geräteaufbau und Grundeinstellung kurz zusammengefasst sind. Entscheidend für die Akzeptanz der NIV ist dabei das Wohlbefinden der Patienten. Dies rechtfertigt den personellen Aufwand einer engmaschigen Kontrolle durch Ärzte und Pflegepersonal.

\section{Fazit für die Praxis}

Die nicht-invasive Beatmung stellt eine zusätzliche therapeutische Option auf der Intensivstation dar, die eine Intubation vermeiden oder die Intubationsdauer verkürzen kann. Wahrscheinlich ist eine Reduktion der mit der Beatmung assoziierten Komplikationen möglich. Initial ist eine gewisse Einarbeitung in die Technik notwendig, um therapeutische Erfolge zu erzielen. Der einfachste Weg zur Erlernung der Technik ist die Hospitation in Kliniken, in denen die nicht-invasive Beatmung bereits eingesetzt wird. 


\section{Literatur}

1. Antonelli M, Conti G, Rocco M et al. (1998) A comparison of noninvasive positive-pressure ventilation and conventional mechanical ventilation in patients with acute respiratory failure. N Engl J Med 339:429-435

2. Barach AL (1935) The use of helium in the treatment of asthma and obstructive lesions in the larynx and trachea. Ann Intern Med 9:739-765

3. Barach AL, Martin J, Eckman M (1938) Positivepressure respiration and its application to the treatment of acute pulmonary edema. Ann Intern Med 12:754-795

4. Benhamou D, Girault C, Faure C, Portier F, Muir JF (1992) Nasal mask ventilation in acute respiratory failure. Experience in elderly patients. Chest 102:912-917

5. Bersten AD, Holt AW, Vedig AE, Skowronski GA, Baggoley CJ (1991) Treatment of severe cardiogenic pulmonary edema with continuous positive airway pressure delivered by face mask. N Engl J Med 325: 1825-1830

6. Bott J,Carroll MP, Conway JH et al. (1993) Randomised controlled trial of nasal ventilation in acute ventilatory failure due to chronic obstructive airways disease. Lancet 341:1555-1557

7. Brett A, Sinclair DG (1993) Use of continuous positive airway pressure in the management of community acquired pneumonia. Thorax 48:1280-1281

8. Brochard L, Isabey D, Piquet J et al. (1990) Reversal of acute exacerbations of chronic obstructive lung disease by inspiratory assistance with a face mask. N Engl J Med 323:1523-1530

9. Brochard L, Mancebo J, Wysocki M et al. (1995) Noninvasive ventilation for acute exacerbations of chronic obstructive pulmonary disease. N Engl J Med 333:817-822

10. Bunburaphong $T$, Imanaka $H$, Nishimura $M$, Hess D, Kacmarek RM (1997) Performance characteristics of bilevel pressure ventilators: a lung model study. Chest 111:1050-1060

11. Confalonieri M, Aiolfi S, Scartabellati A, Parigi $P$, Patrini P, Ghio L, Gandola L (1995) Use of non invasive positive pressure ventilation in severe community-acquired pneumonia.

Am J Respir Crit Care Med 151:A424

12. Confalonieri M, Potena A, Carbone G, Porta RD, Tolley EA, Umberto Meduri G (1999) Acute respiratory failure in patients with severe community-acquired pneumonia. A prospective randomized evaluation of noninvasive ventilation. Am J Respir Crit Care Med 160: 1585-1591

13. Covelli HD, Weled BJ, Beekman JF (1982) Efficacy of continuous positive airway pressure administered by face mask. Chest 81:147-150

14. DeVita MA, Friedman Y, Petrella V (1993) Mask continuous positive airway pressure in AIDS. Crit Care Clin 9:137-151
15. Diaz O, Iglesia R, Ferrer M et al. (1997) Effects of noninvasive ventilation on pulmonary gas exchange and hemodynamics during acute hypercapnic exacerbations of chronic obstructive pulmonary disease. Am J Respir Crit Care Med 156: 1840-1845

16. Elliott MW, Steven MH, Phillips GD, Branthwaite MA (1990) Non-invasive mechanical ventilation for acute respiratory failure. BMJ 300:358-360

17. Ferguson GT, Gilmartin $\mathrm{M}$ (1995) $\mathrm{CO}_{2}$ rebreathing during BiPAP ventilatory assistance. Am J Respir Crit Care Med 151:1126-1135

18. Fernandez $R$, Blanch L, Valles J, Baigorri F, Artigas A (1993) Pressure support ventilation via face mask in acute respiratory failure in hypercapnic COPD patients. Intensive Care Med 19:456-461

19. Foglio C, Vitacca M, Quadri A, Scalvini S, Marangoni S, Ambrosino N (1992) Acute exacerbations in severe COLD patients. Treatment using positive pressure ventilation by nasal mask. Chest 101:1533-1538

20. Gachot B, Clair B, Wolff M, Regnier B, Vachon F (1992) Continuous positive airway pressure by face mask or mechanical ventilation in patients with human immunodeficiency virus infection and severe Pneumocystis carinii pneumonia. Intensive Care Med 18:155-159

21. Girault C, Daudenthun I, Chevron V, Tamion F, Leroy J, Bonmarchand G (1999) Noninvasive ventilation as a systematic extubation and weaning technique in acute-on-chronic respiratory failure: a prospective, randomized controlled study. Am J Respir Crit Care Med 160:86-92

22. Greenbaum DM, Millen JE, Eross B, Snyder JV, Grenvik A, Safar P (1976) Continuous positive airway pressure without tracheal intubation in spontaneously breathing patients. Chest 69:615-620

23. Gregg RW, Friedman BC, Williams JF, McGrath BJ, Zimmerman JE (1990) Continuous positive airway pressure by face mask in Pneumocystis carinii pneumonia. Crit Care Med 18:21-24

24. Hodson ME, Madden BP, Steven MH, Tsang VT, Yacoub MH (1991) Non-invasive mechanical ventilation for cystic fibrosis patients a potential bridge to transplantation. Eur Respir J 4:524-527

25. Hoffmann B, Welte T (1999) The use of noninvasive pressure support ventilation for severe respiratory insufficiency due to pulmonary oedema. Intensive Care Med 25:15-20

26. Keenan SP, Kernerman PD, Cook DJ, Martin CM, McCormack D, Sibbald WJ (1997) Effect of noninvasive positive pressure ventilation on mortality in patients admitted with acute respiratory failure: a meta-analysis. Crit Care Med 25:1685-1692

27. Kilger E, Briegel J, Haller M et al. (1999) Effects of noninvasive positive pressure ventilatory support in non-COPD patients with acute respiratory insufficiency after early extubation. Intensive Care Med 25:1374-1380

28. Köhler D, Schönhofer B (1994) Weaning nach Langzeitbeatmung bei Patienten mit erschöpfter Atempumpe - ein neues Behandlungskonzept. Med Klinik 89:11-15
29. Laier-Groeneveld G, Kupfer J, Hüttemann U, Crièe CP (1992) Weaning from invasive mechanical ventilation. Am Rev Respir Dis 145: A518

30. L'Her E, Duquesne F, Paris A, Mouline J, Renault A, Garo B, Boles JM (1998) Ventilation spontanee en pression expiratoire positive au cours de l'oedeme pulmonaire cardiogenique de la personne agee. Etude dans un service d'accueil et d'urgence. Presse Med 27: 1089-1094

31. Lorenz J, Friesecke S, Huntemann M, Kelbel C (2000) Die nichtinvasive Beatmung in der Beatmungsentwöhnung. Pneumologie 54:10-15

32. Meduri GU (1996) Noninvasive positive-pressure ventilation in patients with acute respiratory failure. Clin Chest Med 17:513-553

33. Meduri GU, Abou-Shala N, Fox RC, Jones CB, Leeper KV, Wunderink RG (1991) Noninvasive face mask mechanical ventilation in patients with acute hypercapnic respiratory failure. Chest 100:445-454

34. Meduri GU, Conoscenti CC, Menashe P, Nair S (1989) Noninvasive face mask ventilation in patients with acute respiratory failure. Chest 95:865-870

35. Meduri GU, Cook TR, Turner RE, Cohen M, Leeper KV (1996) Noninvasive positive pressure ventilation in status asthmaticus. Chest 110:767-774

36. Meduri GU, Turner RE, Abou-Shala N, Wunderink R, Tolley E (1996) Noninvasive positive pressure ventilation via face mask. First-line intervention in patients with acute hypercapnic and hypoxemic respiratory failure. Chest 109:179-193

37. Mehta S, Jay GD, Woolard RH et al. (1997) Randomized, prospective trial of bilevel versus continuous positive airway pressure in acute pulmonary edema. Crit Care Med 25:620-628

38. Nava S, Evangelisti I, Rampulla C, Compagnoni ML, Fracchia C, Rubini F (1997) Human and financial costs of noninvasive mechanical ventilation in patients affected by COPD and acute respiratory failure. Chest 111:1631-1638

39. Pennock BE, Crawshaw L, Kaplan PD (1994) Noninvasive nasal mask ventilation for acute respiratory failure. Institution of a new therapeutic technology for routine use. Chest 105:441-444

40. Pennock BE, Kaplan PD, Carlin BW, Sabangan JS, Magovern JA (1991) Pressure support ventilation with a simplified ventilatory support system administered with a nasal mask in patients with respiratory failure. Chest 100:1371-1376

41. Piper AJ, Parker S, Torzillo PJ, Sullivan CE, Bye PT (1992) Nocturnal nasal IPPV stabilizes patients with cystic fibrosis and hypercapnic respiratory failure. Chest 102:846-850

42. Pollack C, Torres MT, Alexander L (1996) Feasibility study of the use of bilevel positive airway pressure for respiratory support in the emergency department. Ann Emerg Med 27:189-192

43. Rabbat A, Leleu G, Bekka F et al. (1995) Non invasive ventilation in HIV patients with severe Pneumocystis carinii pneumonia. Am J Respir Crit Care Med 151:A427 
44. Rasanen J, Heikkila J, Downs J, Nikki P, Vaisanen I, Viitanen A (1985) Continuous positive airway pressure by face mask in acute cardiogenic pulmonary edema. Am J Cardiol 55:296-300

45. Rusterholtz T, Kempf J, Berton C et al. (1999) Noninvasive pressure support ventilation (NIPSV) with face mask in patients with acute cardiogenic pulmonary edema (ACPE). Intensive Care Med 25:21-28

46. Schönhofer B, Sonneborn M, Haidl P, Kemper K, Köhler D (1995) Stellenwert der intermittierenden Selbstbeatmung nach Entwöhnung vom Respirator. Pneumologie 49:689-694

47. Sen N, Dhanraj P (1994) Use of facemask continuous positive airway pressure (CPAP) in patients with refractory hypoxaemia caused by burn sepsis. Burns 20:271-272

48. Servera E, Perez M, Marin J,Vergara P, Castano R (1995) Noninvasive nasal mask ventilation beyond the ICU for an exacerbation of chronic respiratory insufficiency. Chest 108: 1572-1576

49. Shivaram U, Donath J, Khan FA, Juliano J (1987) Effects of continuous positive airway pressure in acute asthma. Respiration 52:157-162

50. Smith RA, Kirby RR, Gooding JM, Civetta JM (1980) Continuous positive airway pressure (CPAP) by face mask. Crit Care Med 8:483-485

51. Suter PM, Kobel N (1981) Treatment of acute pulmonary failure by CPAP via face mask: when can intubation be avoided? Klin Wochenschr 59:613-616

52. Udwadia ZF, Santis GK, Steven MH, Simonds AK (1992) Nasal ventilation to facilitate weaning in patients with chronic respiratory insufficiency. Thorax 47:715-718

53. Vitacca M, Clini E, Rubini F, Nava S, Foglio K, Ambrosino N (1996) Non-invasive mechanical ventilation in severe chronic obstructive lung disease and acute respiratory failure: shortand long-term prognosis. Intensive Care Med 22:94-100

54. Wysocki M, Tric L, Mazeyrac C, Wolff M, Gertner J, Millet H, Herman B (1992) Non invasive pressure support ventilation (NIPSV) in acute respiratory failure (ARF). Am Rev Respir Dis 145:A527

55. Wysocki M, Tric L, Wolff MA, Millet H, Herman B (1995) Noninvasive pressure support ventilation in patients with acute respiratory failure. A randomized comparison with conventional therapy. Chest 107:761-768 